\title{
Status of the NA62 Experiment
}

\author{
M. Piccini ${ }^{1, a, b}$ \\ ${ }^{1}$ INFN - Sezione di Perugia
}

\begin{abstract}
The decay $K^{+} \rightarrow \pi^{+} v \bar{v}$, with a very precisely predicted branching ratio of less than $10^{-10}$ in the Standard Model framework, is one of the best candidates to reveal indirect effects of new physics at the highest mass scales. The NA62 experiment at CERN SPS is designed to measure the branching ratio of such decay with a decay-in-flight technique, novel for this channel. The main goal of NA62 is to measure such Branching Ratio of $K^{+} \rightarrow \pi^{+} v \bar{v}$ with an accuracy better than $20 \%$. This will be achieved by collecting up to $100 \mathrm{~K}^{+} \rightarrow \pi^{+} v \bar{v}$ events with a background contamination at the level of 10\%. The NA62 detector was commissioned in 2014 and 2015 and the experiment took physics data from 2015 to 2018 . NA62 has already published the result of the 2016 data analysis. The 2017 data analysis is in progress, the single event sensitivity reached and the evaluation of the main backgrounds will be shown in this contribution.
\end{abstract}

\section{Introduction}

The $K^{+} \rightarrow \pi^{+} v \bar{v}$ is a decay proceeding through box and electroweak penguin diagrams. A quadratic GIM mechanism and the transition of the quark strange into the quark

\footnotetext{
${ }^{\mathrm{a}} \mathrm{e}$-mail: Mauro.Piccini@pg.infn.it

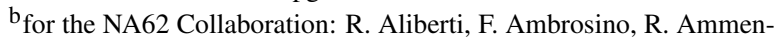
dola, B. Angelucci, A. Antonelli, G. Anzivino,R. Arcidiacono, T. Bache, M. Barbanera, J. Bernhard, A. Biagioni, L. Bician, C. Biino, A. Bizzeti, T. Blazek, B. Bloch-Devaux, V. Bonaiuto, M. Boretto, M. Bragadireanu, D. Britton, F. Brizioli, M.B. Brunetti, D. Bryman, F. Bucci,T. Capussela, J. Carmignani, A. Ceccucci, P. Cenci, V. Cerny, C. Cerri, B. Checcucci, A. Conovaloff, P. Cooper, E. CortinaGil, M. Corvino, F. Costantini, A. Cotta Ramusino, D. Coward, G. D'Agostini, J. Dainton, P. Dalpiaz, H. Danielsson, N. DeSimone, D. Di Filippo, L. Di Lella, N. Doble, B. Dobrich, F. Duval, V. Duk, J. Engelfried, T. Enik, N. Estrada-Tristan,V. Falaleev, R. Fantechi, V. Fascianelli, L. Federici, S. Fedotov, A. Filippi, M. Fiorini, J. Fry, J. Fu, A. Fucci, L. Fulton,E. Gamberini, L. Gatignon, G. Georgiev, S. Ghinescu, A. Gianoli, M. Giorgi, S. Giudici, F. Gonnella, E. Goudzovski,C. Graham, R. Guida, E. Gushchin, F. Hahn, H. Heath, E.B. Holzer, T. Husek, O. Hutanu, D. Hutchcroft, L. Iacobuzio,E. Iacopini, E. Imbergamo, B. Jenninger, J. Jerhot, R.W. Jones, K. Kampf, V. Kekelidze, S. Kholodenko, G. Khoriauli,A. Khotyantsev, A. Kleimenova, A. Korotkova, M. Koval, V. Kozhuharov, Z. Kucerova, Y. Kudenko, J. Kunze, V. Kurochka,V. Kurshetsov, G. Lanfranchi, G. Lamanna, E. Lari, G. Latino, P. Laycock, C. Lazzeroni, M. Lenti, G. Lehmann Miotto,E. Leonardi, P. Lichard, L. Litov, R. Lollini, D. Lomidze, A. Lonardo, P. Lubrano, M. Lupi, N. Lurkin, D. Madigozhin,I. Mannelli, G. Mannocchi, A. Mapelli, F. Marchetto, R. Marchevski, S. Martellotti, P. Massarotti, K. Massri, E. Maurice,M. Medvedeva, A. Mefodev, E. Menichetti, E. Migliore, E. Minucci, M. Mirra, M. Misheva, N. Molokanova, M. Moulson,S. Movchan, M. Napolitano, I. Neri, F. Newson, A. Norton, M. Noy, T. Numao, V. Obraztsov, A. Ostankov, S. Padolski,R. Page, V. Palladino, A. Parenti, C. Parkinson, E. Pedreschi, M. Pepe, M. PerrinTerrin, L. Peruzzo, P. Petrov,Y. Petrov, F. Petrucci, R. Piandani, M. Piccini, J. Pinzino, I. Polenkevich, L. Pontisso, Yu. Potrebenikov, D. Protopopescu,M. Raggi, A. Romano, P. Rubin, G. Ruggiero, V. Ryjov, A. Salamon, C. Santoni, G. Saracino, F. Sargeni, S. Schuchmann,V. Semenov, A. Sergi, A. Shaikhiev, S. Shkarovskiy, D. Soldi, V. Sugonyaev, M. Sozzi, T. Spadaro, F. Spinella, A. Sturgess,J. Swallow, S. Trilov, P. Valente, B. Velghe, S. Venditti, P. Vicini, R. Volpe, M. Vormstein, H. Wahl, R. Wanke, B. Wrona,O. Yushchenko, M. Zamkovsky, A. Zinchenko.
}

down (flavour changing neutral current) make this process extremely rare. The Standard Model predicts [1] the branching ratio to be $(8.4 \pm 1.0) \times 10^{-11}$, where the precision on the external inputs (measurable parameters) dominates the uncertainty. The theoretical accuracy, instead, is at the level of $2 \%$ [2].

On the other hand the $K^{+} \rightarrow \pi^{+} v \bar{v}$ decay is extremely sensitive to physics beyond the SM, probing the highest mass scales among the rare meson decays. The largest deviations from SM are expected in models with new sources of flavour violation [3, 4]. Depending on new physics models, correlation patterns between possible deviations from the SM prediction of $K^{+} \rightarrow \pi^{+} v \bar{v}$ and $K_{L} \rightarrow \pi^{0} v \bar{v}$ decay modes are expected [5]. The $K^{+} \rightarrow \pi^{+} v \bar{v}$ decay can also be sensitive to effects of lepton flavour non-universality [6] and can constrain leptoquark models [7]. Present experimental constraints limit the range of variation within supersymmetric models [810].

The experiments E787 and E949 at BNL [11, 12] studied the $K^{+} \rightarrow \pi^{+} v \bar{v}$ decay using a kaon decay-at-rest technique, their measured Branching Ratio is $\left(17.3_{-10.5}^{+11.5}\right) \times$ $10^{-11}$

NA62 has collected data with a good quality for the measurement of $K^{+} \rightarrow \pi^{+} v \bar{v}$ from 2016 to 2018. The result of the data collected in 2016 [13], with one event found in the signal region $(0.26$ events expected for the Standard Model decay and with an expected background contribution of 0.12 events) allowed to set a $95 \%$ confidence level limit on the branching ratio: $\mathrm{BR}\left(K^{+} \rightarrow\right.$ $\left.\pi^{+} v \bar{v}\right)<14 \times 10^{-10}$ and demonstrated the validity of this experimental technique. The 2017 data analysis is in progress. In the following sections, after the description of the beam line and of the experimental setup, the selection and reconstruction of the signal, the measurement of 


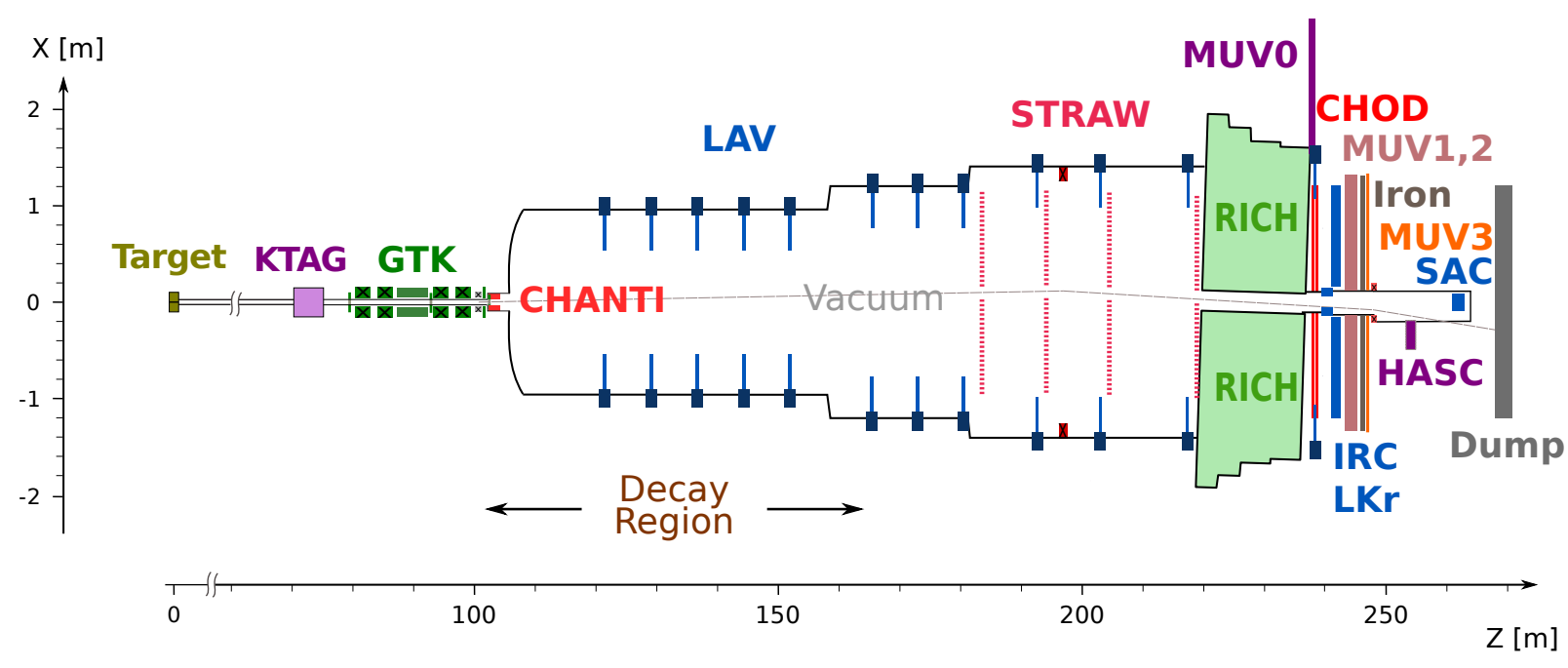

Figure 1. Schematic view of the NA62 experimental setup.

the Kaon flux, the evaluation of the single event sensitivity and the contribution of most of the backgrounds will be presented.

\section{The NA62 beam and detector}

NA62 is the last generation kaon experiment at CERN SPS aiming to study the decay $K^{+} \rightarrow \pi^{+} v \bar{v}$. The goal of the experiment is to measure the decay branching ratio with at least a $20 \%$ accuracy, collecting up to 100 events in few years of data taking. The $400 \mathrm{GeV} / \mathrm{c}$ proton beam extracted from the SPS in the north area at CERN meets such demanding request in terms of intensity by providing $2 \cdot 10^{12}$ proton/spill (effective spill length $\sim 3 \mathrm{~s}$ ). Such protons beam impinges on a beryllium target (for a total of $\sim 10^{18}$ protons on target per year) from which a secondary charged hadron beam of $75 \mathrm{GeV} / \mathrm{c}$ and $1 \%$ momentum bite is selected and transported downstream. About $6 \%$ of beam particles are $K^{+}$.

The NA62 detector must be able to reject background events from decay channels with branching ratios up to 10 orders of magnitude higher than the signal and with similar experimental signature, such as $K^{+} \rightarrow \mu^{+} v$ and $K^{+} \rightarrow \pi^{+} \pi^{0}$. To this purpose, good kinematic rejection, particle identification (PID) and veto systems are required. A schematic view of the experimental setup is shown in Fig. 1.

The required resolution on the measurement of kinematic parameters (momenta, missing mass) is obtained tracking both the decaying kaon with a silicon pixel detector (GTK) and the parent charged particles with a straw spectrometer (STRAW). The key elements of the PID in NA62 are: a differential Cherenkov detector (KTAG) to identify kaons in the charged beam, a Ring Imaging Cherenkov detector (RICH) to identify $\pi^{+}$and $\mu^{+}$ in the momentum range between 15 and $35 \mathrm{GeV} / \mathrm{c}$ and three scintillator detectors acting as hadron calorimeters (MUV1, MUV2) and muon counter (MUV3) at the end of the detector region. High resolution on time measurement is also required in order to reduce background produced by event overlaps. Large Photon Vetos (LAV) made by lead glass ad large angle, a Liquid Krypton calorimeter (LKr) at intermediate angles and two shashlik detectors at small angles (IRC, SAC) are mainly used to suppress background from kaon decays with photons in the final state. The experimental setup is completed by a scintillator detector (CHANTI) at the beginning of the fiducial decay region in order to reduce critical background induced by inelastic interactions of the beam with the collimator and the GTK and by an hadron sampling calorimeter (HASC) to reduce the background from kaon decays with particle products traveling along the beam line. A detailed description of the beam and of the experimental setup can be found here [14].

The detectors have to stand a rate of about $10 \mathrm{MHz}$ of events, high performances read-out and trigger systems have been developed for this purpose [15].

\section{Analysis strategy and signal selection}

The signature of a $K^{+} \rightarrow \pi^{+} v \bar{v}$ decay is one $K^{+}$in the initial state and one $\pi^{+}$associated to missing energy in the final state. The main kinematic variable is $m_{m i s s}^{2}=$ $\left(P_{K}-P_{\pi}\right)^{2}$, where $P_{K}$ and $P_{\pi}$ are the 4-momenta of the $K^{+}$and $\pi^{+}$, respectively. The $m_{\text {miss }}^{2}$ of the signal is broadly distributed, as shown in Fig. 2. Search for signal occurs in two regions of the $m_{\text {miss }}^{2}$ spectrum surrounding the peak of the $K^{+} \rightarrow \pi^{+} \pi^{0}$ at the $\pi^{0}$ mass. Possible backgrounds are:

- $K^{+} \rightarrow \pi^{+} \pi^{0}, K^{+} \rightarrow \mu^{+} v$ and $K^{+} \rightarrow \pi^{+} \pi^{+} \pi^{-}$entering signal regions through non gaussian resolution and radiative tails of the $m_{m i s s}^{2}$ ditribution

- $K^{+} \rightarrow \pi^{0} l v_{l}(l=e, \mu)$ decays and more rare processes like $K^{+} \rightarrow \pi^{+} \pi^{-} e^{+} v$ broadly distributed across the signal regions

- Events mimicking $K^{+} \rightarrow \pi^{+} v \bar{v}$ originating along the beam line via inelastic interactions of beam particles with the material 


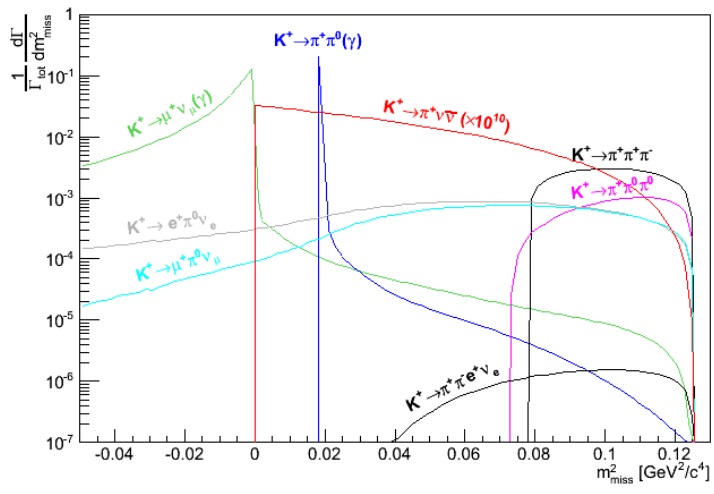

Figure 2. $m_{\text {miss }}^{2}$ in the $\pi^{+}$mass hypothesis for the positive particle for signal and other $\mathrm{K}^{+}$decays.

- $\mathrm{K}^{+}$decaying before entering the fiducial volume downstream to the last station of the GTK (GTK3)

Each source of background requires different rejection procedures, depending on the kinematics and on the type and number of charged particles in the final state. The estimation of the expected background remaining after selection is done separately for each process. A blind analysis procedure has been adopted, with signal and control regions that will be kept masked until completion of the selection criteria and the evaluation of the expected number of signal and background events.

Selection criteria and reconstruction proceed with the following steps. A positive track reconstructed in the STRAW and associated to signals in RICH, $\mathrm{LKr}$ and CHOD defines a $\pi^{+}$. A signal in the KTAG associated to a GTK track defines a $K^{+}$. The $K^{+}$is coupled to the $\pi^{+}$ by matching in time and space the corresponding tracks. Selection criteria applied to the reconstructed decay vertex define the fiducial decay region downstream of the last GTK station. In addition events are rejected if the $\pi^{+}$track originates from the beam aperture of the final collimator.

The analysis is restricted to $(15,35) \mathrm{GeV} / \mathrm{c} \pi^{+}$momentum, leaving at least $40 \mathrm{GeV}$ of extra energy to veto background events from other $\mathrm{K}^{+}$decays. The selection proceeds with the $\pi^{+}$identification using the calorimeters and the RICH. Eventually events with muons are suppressed by 8 orders of magnitude. Events with photons are rejected by exploiting the time coincidence between the $\pi^{+}$and possible signals in LAV, LKr, IRC or SAC. Selection criteria based on CHOD's and STRAW's activity are employed against photons interacting with the material upstream of the LKr. The $\pi^{0}$ detection inefficiency is about $1.4 \times 10^{-8}$, measured on data. The above criteria are also effective against decays like $K^{+} \rightarrow \pi^{+} \pi^{+} \pi^{-}$and $K^{+} \rightarrow \pi^{+} \pi^{-} e^{+} \nu$. Finally events in presence of CHANTI signals in time with the $\pi^{+}$or extra hits in at least 2 GTK stations are rejected. The $K^{+} \rightarrow \pi^{+} v \bar{v}$ candidates are those events in regions 1 and 2 that pass the selection criteria described above.

Fig. 3 shows the distribution of the selected events in the $\left(m_{\text {miss }}^{2}, p_{\pi^{+}}\right)$plane, where $p_{\pi^{+}}$is the $\pi^{+}$momentum. The $m_{m i s s}^{2}$ is computed using the $K^{+}$and $\pi^{+}$momenta measured in GTK and STRAW assuming the $K^{+}$and $\pi^{+}$masses, respectively. In this plane two signal regions are defined, termed Signal Region 1 and 2, and three background regions mostly populated by $K^{+} \rightarrow \pi^{+} \pi^{0}, K^{+} \rightarrow \mu^{+} v$ and $K^{+} \rightarrow \pi^{+} \pi^{+} \pi^{-}$decays.

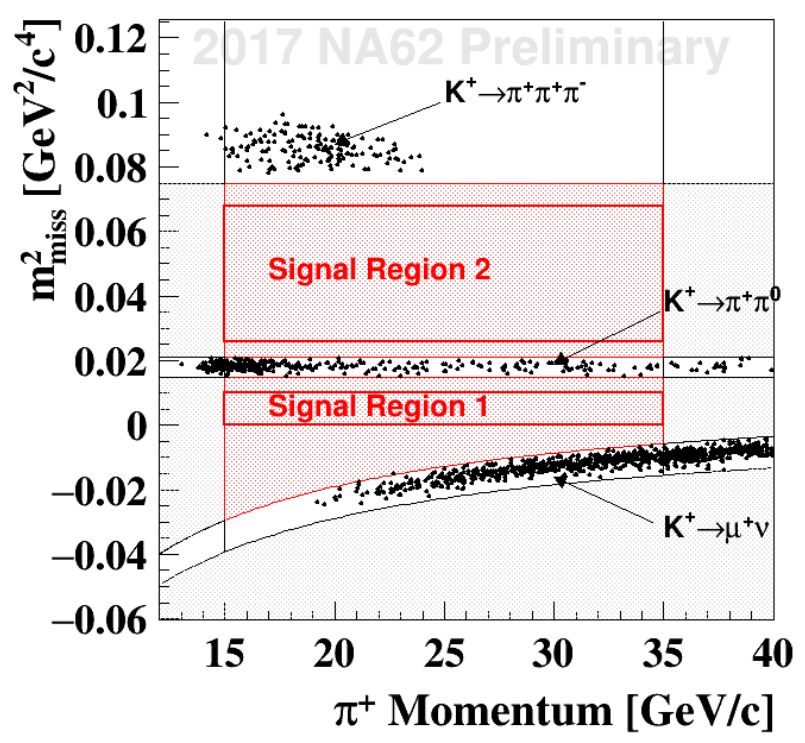

Figure 3. $m_{\text {miss }}^{2}$ as a function of $p_{\pi^{+}}$) after $K^{+} \rightarrow \pi^{+} v \bar{v}$ selection. The two signal regions and their surrounding regions (control regions) are still blind.

\section{Kaon flux and single event sensitivity}

The number of expected $K^{+} \rightarrow \pi^{+} v \bar{v}$ events in the Standard Model hypothesis, $N_{\pi v v}^{S M}$, is estimated as:

$$
N_{\pi v v}^{S M}=N_{\pi^{+} \pi^{0}} \epsilon_{\text {trigger }} \epsilon_{R V} \frac{A_{\pi v v}}{A_{\pi^{+} \pi^{0}}} \frac{B R^{S M}(\pi v v)}{B R\left(\pi^{+} \pi^{0}\right)}
$$

where $N_{\pi^{+} \pi^{0}}$ is the number of $K^{+} \rightarrow \pi^{+} \pi^{0}$ decays selected in 2017 with a minimum bias trigger, $\epsilon_{\text {trigger }}$ and $\epsilon_{R V}$ are the trigger and the random veto efficiencies for the $\pi v v$ selection, $A_{\pi v v}$ and $A_{\pi^{+} \pi^{0}}$ are the geometrical and selection acceptances for signal and for the normalization channel $K^{+} \rightarrow \pi^{+} \pi^{0}$ estimated from Monte Carlo simulations and $B R^{S M}(\pi v v)$ and $B R\left(\pi^{+} \pi^{0}\right)$ are the the predicted SM branching ratio of the $K^{+} \rightarrow \pi^{+} v \bar{v}$ decay and the measured branching ration of the $K^{+} \rightarrow \pi^{+} \pi^{0}$ decay, respectively. For the selection of the $K^{+} \rightarrow \pi^{+} \pi^{0}$ candidates the same offline $\pi v v$ selection criteria are used, excluding the photon rejection cuts and requiring the $m_{\text {miss }}^{2}$ in the $K^{+} \rightarrow \pi^{+} \pi^{0}$ region (at the $\pi^{0}$ mass) . $\epsilon_{\text {trigger }}$ and $\epsilon_{R V}$ are both measured on data. In particular $\epsilon_{R V}$ is defined as one minus the probability for a signal event to be randomly rejected by the photon veto conditions and it is measured to be about $64 \%$ on average. Both $\epsilon_{\text {trigger }}$ and $\epsilon_{R V}$ depends on the instantaneous beam intensity and they are combined taking into account such dependence. The activity in the time sidebands of the GTK allows for the estimation of the instantaneous beam intensity event-by-event. 
The number $N_{\pi v v}$ is evaluated in four bins of $\pi^{+}$momentum. By definition, the single event sensitivity (SES) is equal to $B R^{S M}(\pi v v) / N_{\pi v v}^{S M}$. In the 2017 sample $N_{\pi v v}^{S M}=$ $2.5 \pm 0.4$ corresponding to a single event sensitivity (SES) of $(0.34 \pm 0.04) \times 10^{-10}$. The uncertainty on the SES is systematical and mostly stems from the evaluation of $\epsilon_{\text {trigger }}$ and $A_{\pi v v}$. The uncertainty on $N_{\pi v v}^{S M}$ is dominated by the theoretical error on the value of $B R^{S M}(\pi v v)$. The achieved sensitivity corresponds to about $1.3 \times 10^{12} K^{+}$decaying in the fiducial decay volume during the 2017 run (kaon flux).

\section{Background evaluation}

A class of backgrounds that has been already estimated for the 2017 sample is the one originating from $K^{+}$decaying in the fiducial volume. In particular, the estimation of the $K^{+} \rightarrow \pi^{+} \pi^{0}$ and $K^{+} \rightarrow \mu^{+} v$ backgrounds (including their radiative components) relies on the assumption that $\pi^{0}$ and $\mu^{+}$rejections are independent from the $m_{\text {miss }}^{2}$ cuts defining the signal regions. Kinematically unbiased samples of $K^{+} \rightarrow \pi^{+} \pi^{0}$ and $K^{+} \rightarrow \mu^{+} v$ events selected on minimum bias data provide a measurement of the probability for these decays to enter the signal regions as a consequence of a mis-reconstructed kinematics. The $K^{+} \rightarrow \pi^{+} \pi^{0}$ sample has been selected tagging the two photons from $\pi^{0}$ decay with the two corresponding clusters in the LKr calorimeter (see Fig. 4).

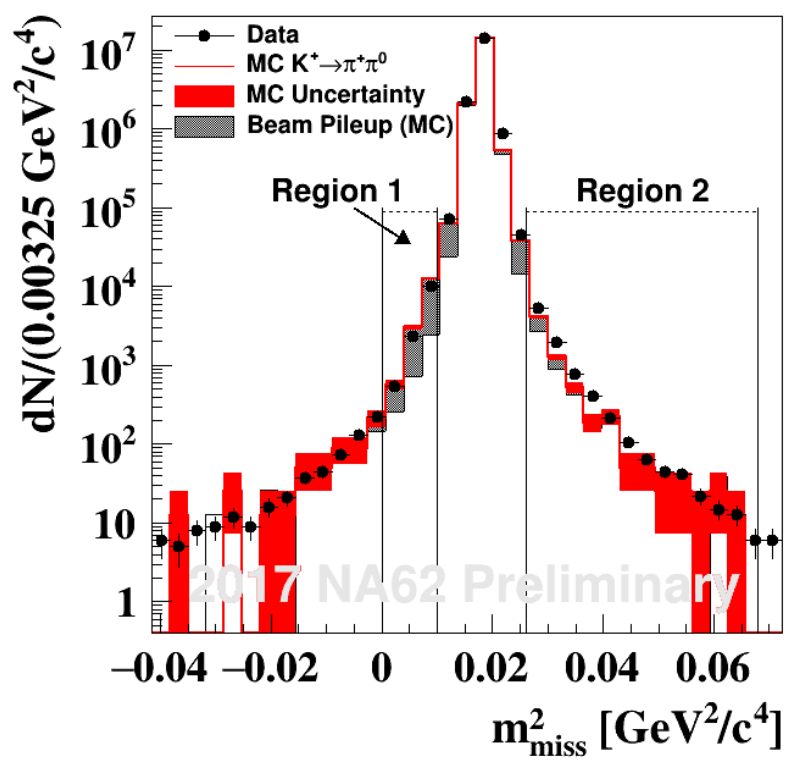

Figure 4. Distribution of $m_{\text {miss }}^{2}$ for a sample of $K^{+} \rightarrow \pi^{+} \pi^{0}$ events.

The $K^{+} \rightarrow \mu^{+} v$ sample has been instead selected tagging the muon with a signal in the MUV3 scintillating detector (see Fig. 5).

The expected background stems from this probability and the number of events surviving the $K^{+} \rightarrow \pi^{+} v \bar{v}$ selection in the corresponding $m_{\text {miss }}^{2}$ background regions. Corrections are applied to account for correlations between kinematics and $\pi^{0}$ or $\mu^{+}$rejections using both simulations

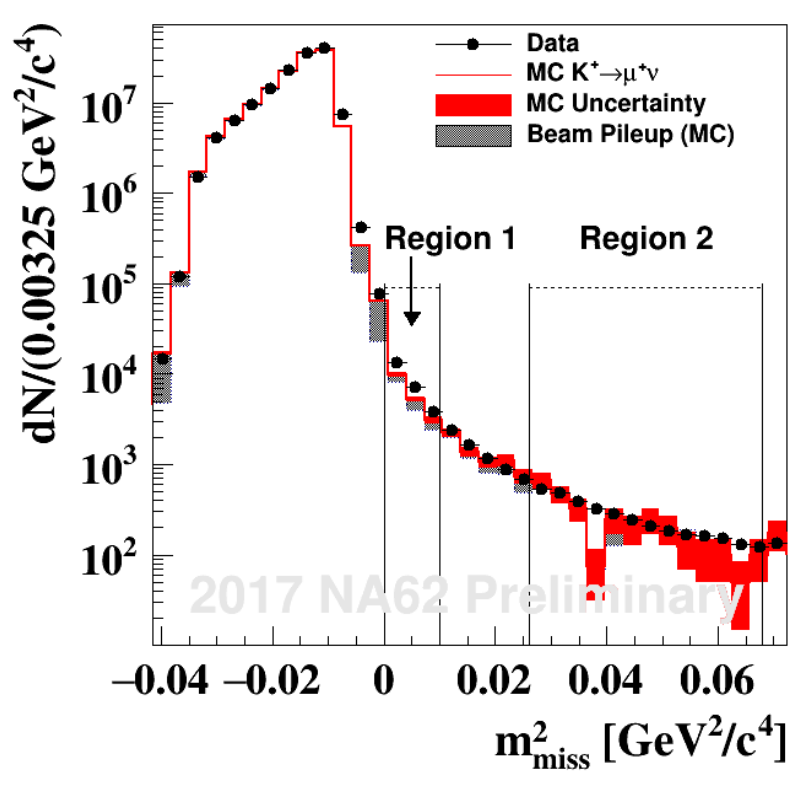

Figure 5. Distribution of $m_{\text {miss }}^{2}$ for a sample of $K^{+} \rightarrow \mu^{+} v$ events.

and data. Comparisons between the expected and observed background events in suitable control regions of the $m_{m i s s}^{2}$ will be used to validate the estimation of these backgrounds. A similar procedure is adopted to assess the $K^{+} \rightarrow \pi^{+} \pi^{+} \pi^{-}$background. The estimation of the other backgrounds from $K^{+}$decays relies on Monte Carlo simulations normalized to $K^{+} \rightarrow \pi^{+} \pi^{0}$ decays, as for the signal. Samples of background-enriched data are used to validate the simulation down to the $K^{+} \rightarrow \pi^{+} v \bar{v}$ single event sensitivity. As an example, in Fig. 6 is shown the distribution of $m_{m i s s}^{2}$ for Monte Carlo $K^{+} \rightarrow \pi^{+} \pi^{-} e^{+} v$ events once the multiplicity criteria applied in the $\pi v v$ selection have been inverted in order to have a sizeble sample of the same type of events even in data (comparison used to validate the Monte Carlo).

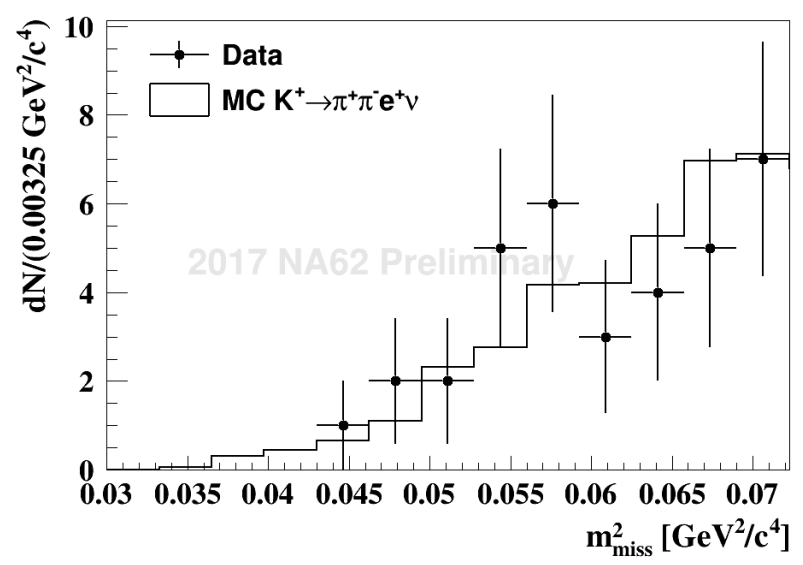

Figure 6. Distribution of $m_{m i s s}^{2}$ for a Monte Carlo sample of $K^{+} \rightarrow \pi^{+} \pi^{-} e^{+} v$ decays.

Upstream background events are $\pi^{+} \mathrm{s}$ originated from decays or interactions of $\mathrm{K}^{+} \mathrm{s}$ occurring upstream of the 
Table 1. Expected number of signal and background events in the signal regions

\begin{tabular}{ll}
\hline Process & Expected events in signal regions \\
\hline$K^{+} \rightarrow \pi^{+} v \bar{v}$ & $2.5 \pm 0.4$ \\
\hline$K^{+} \rightarrow \pi^{+} \pi^{0}(\gamma)$ & $0.35 \pm 0.02_{\text {stat }} \pm 0.03_{\text {syst }}$ \\
$K^{+} \rightarrow \mu^{+} v(\gamma)$ & $0.16 \pm 0.01_{\text {stat }} \pm 0.05_{\text {syst }}$ \\
$K^{+} \rightarrow \pi^{+} \pi^{-} e^{+} v$ & $0.22 \pm 0.08_{\text {stat }}$ \\
$K^{+} \rightarrow \pi^{+} \pi^{+} \pi^{-}$ & $0.015 \pm 0.008_{\text {stat }} \pm 0.015_{\text {syst }}$ \\
$K^{+} \rightarrow \pi^{0} l v_{l}$ & $0.012 \pm 0.012_{\text {syst }}$ \\
$K^{+} \rightarrow \pi^{+} \gamma \gamma$ & $0.005 \pm 0.005_{\text {syst }}$ \\
\hline
\end{tabular}

final collimator. In these cases a lone $\pi^{+}$can enter the region downstream through the beam aperture of the final collimator, with the collimator itself preventing the detection of the extra energy produced concurrently with such $\pi^{+}$. The $\pi^{+}$mimics a signal event if accidentally matched to a random beam particle. In addition the direction of the $\pi^{+}$must be mis-reconstructed to create a fake decay vertex in the fiducial volume and to trace back the $\pi^{+}$outside the beam aperture of the final collimator. The estimation of upstream background (the main source of background on 2016 result) on the 2017 sample is still ongoing.

In Table 1 are reported the contributions of the main backgrounds to the $K^{+} \rightarrow \pi^{+} v \bar{v}$ selection as estimated up to now.

\section{Conclusions}

The analysis $K^{+} \rightarrow \pi^{+} v \bar{v}$ on the data collected during the 2017 run by the NA62 experiment will be soon finalized. Up to now the expected number of events in the 2017 sample is about a factor 10 higher than the one expected in 2016, with a similar signal/background ratio. One of the main background to the measurement is the upstream background described above. To better suppress this type of background in 2018 a new collimator has been installed at the beginning of the fiducial decay volume. The analysis of 2018 sample will start as soon as the 2017 analysis will be completed.

NA62 has already been approved to take data in 2021, after the end of SPS and LHC two years long shut down. This new data taking period, that will probably be extended until the next SPS and LHC long shut down in
2024, will allow to further increase the collected sample of $K^{+} \rightarrow \pi^{+} v \bar{v}$ decays.

The NA62 collaboration is also active in several other analyses and recently new results have been published on the search for dark photons on $\pi^{0}$ decays [16] and on the search for lepton number violation on $K^{+}$decays with two leptons in the final state [17].

\section{References}

[1] Buras A. J., Buttazzo D., Girrbach-Noe J. and Knegjens R., JHEP 1511033 (2015)

[2] Brod J., Gorbahn M. and Stamou E., Phys. Rev. D 83 034030 (2011)

[3] Blanke M., Buras A. J. and Recksiegel S., Eur. Phys. J. C 76 no.4 182 (2016)

[4] Blanke M., Buras A. J., Duling B., Gemmler K. and Gori S. JHEP 0903108 (2009)

[5] Buras A. J., Buttazzo D. and Knegjens R., JHEP 1511 166 (2015)

[6] Isidori G., Mescia F., Paradisi P., Smith C. and Trine S., JHEP 0608064 (2006)

[7] Blazek T. and Matak P., Nucl. Phys. Proc. Suppl. 198 216 (2010)

[8] Tanimoto M. and Yamamoto K., PTEP 20162016 no.12 123B02 (2016)

[9] Bordone M., Buttazzo D., Isidori G. and Monnard J., Eur. Phys. J. C 77 no.9 618 (2017)

[10] Bobeth C. and Buras A. J., JHEP 1802101 (2018)

[11] Artamonov A. V. et al., Phys. Rev. Lett. 101191802 (2008)

[12] Artamonov A. V. et al., Phys. Rev. D 79092004 (2008)

[13] Cortina Gil E. et al., Phys. Lett. B 791 156-66. (2019)

[14] Cortina Gil E. et al., J. Instrum. 12 P05025 (2017)

[15] Ammendola R. et al., Nucl. Instrum. Meth. A 929 001-022 (2019)

[16] Cortina Gil E. et al., JHEP 1905182 (2019)

[17] Cortina Gil E. et al., Phys.Lett. B 797134794 (2019) 